\title{
The Exceptional Jordan Algebra and the Superstring
}

\author{
E. Corrigan and T. J. Hollowood \\ Department of Mathematical Science, Science Laboratories, South Road, Durham DH1 3LE, UK
}

\begin{abstract}
Two representations of the exceptional Jordan algebra are presented, one in terms of bose vertex operators, and the other in terms of superstring vertex operators in bosonised form, including their BRST ghost contributions. It is also shown how the non-exceptional Jordan algebras may be constructed similarly.
\end{abstract}

\section{Introduction}

Over the years it has been suggested that Jordan algebras, which originated in the search for alternative formulations of quantum mechanics [1], might play a rôle in the theory of colour and confinement [2], in supersymmetry [3], and more recently, in the theory of superstrings [4-11]. In particular, physicists have been fascinated by the existence of the unique, exceptional Jordan algebra which may be represented in terms of three dimensional hermitian matrices with octonion elements [12]. In fact Jordan algebras have led to insights more of a mathematical than physical nature [13]. Perhaps string theory will be different, since it already has strong connections with algebras in several ways. In particular, the vertex operators of string theory are able, on the one hand, to represent the couplings of strings, and on the other, to provide certain representations of Lie and Kac-Moody algebras [14-16], and other interesting algebras such as the Virasoro algebra and the algebra associated with the Fischer-Griess monster group [17]. It is therefore natural to ask if there is a connection between vertex operators and Jordan algebras. Indeed, there is a suspicion that there should be a relationship between the exceptional Jordan algebra and the vertex operators of the superstring [5]. Our aim in this article is to make this connection more concrete.

Since our main motivation is to uncover a relationship between the superstring vertices [18-22] and the exceptional Jordan algebra, we shall use this particular example to illustrate our ideas. However, the ideas are certainly extendable to the other Jordan algebras and the relevant details will be explained in Sect. (4).

Abstractly, a Jordan algebra [12] is a real (finite dimensional) vector space $J$ together with a symmetric, but not necessarily associative, vector product satisfying 
the Jordan identity. Thus for any two elements $X, Y$ of $J$ their product is written $X \circ Y$, and is required to satisfy

$$
\begin{gathered}
X \circ Y=Y \circ X, \\
X \circ\left(Y \circ X^{2}\right)=(X \circ Y) \circ X^{2} .
\end{gathered}
$$

Commonly, however, we are interested in representing elements of $J$ by hermitian matrices whose entries are either real, complex, quaternion or octonion. In the latter case, the matrices must be two or three dimensional, while in the other cases the matrices may be of any size. Then, the symmetric product is defined to be the anticommutator

$$
X \circ Y=\frac{1}{2}\{X, Y\}=\frac{1}{2}(X Y+Y X),
$$

and, with the exception of the octonion case, Eq. (1.2) is not restrictive. In the octonian case, Eq. (1.2) restricts the dimension of $J$ as stated.

The exceptional Jordan algebra elements may be regarded as real linear combinations of twenty-seven basis elements. These correspond to the three diagonal units, $e_{\iota}, i=1,2,3$ :

$$
\left(e_{i}\right)_{k l}=\delta_{i k} \delta_{i l}, \quad k, l=1,2 \text { or } 3,
$$

and the twenty-four hermitian matrices, $A_{i j}(a) i, j=1,2,3$, with $i<j$ :

$$
\left(A_{i j}(a)\right)_{k l}=a \delta_{i k} \delta_{j l}+\bar{a} \delta_{i l} \delta_{j k},
$$

where $a$ may be unity, or any one of the octonion imaginary units $u_{i}, i=1, \ldots, 7$. In Eq. (1.5) $\bar{a}$ denotes the octonion conjugate of $a$. It is also convenient to define accompanying matrices for $i>j$ via:

$$
A_{i j}(a)=A_{j i}(\bar{a}) .
$$

In terms of these elements the algebra may be written:

$$
\begin{aligned}
e_{i} \circ e_{j}= & e_{i} \delta_{i j}, \\
e_{i} \circ A_{k l}(a)= & \begin{cases}\frac{1}{2} A_{k l}(a) & i=k \text { or } l \\
0 & \text { otherwise }\end{cases} \\
A_{i j}(a) \circ A_{i j}(b)= & \operatorname{Re}(a \bar{b})\left(e_{i}+e_{j}\right) \quad i<j, \\
A_{i j}(a) \circ A_{k l}(b)= & \frac{1}{2}\left\{\delta_{i k} A_{j l}(\bar{a} b)+\delta_{i l} A_{j k}(\bar{a} \bar{b})\right. \\
& \left.+\delta_{j k} A_{l l}(a b)+\delta_{j l} A_{i k}(a \bar{b})\right\} \quad i<j, k<l .
\end{aligned}
$$

These definitions extend to the other Jordan algebras, for which $i, j=1, \ldots, N$, except that in the cases for which $N>3$ the units may only be chosen from among the real, complex or quaternion numbers.

The twenty-seven basic elements of the exceptional Jordan algebra may be regarded as a 27 of a non-compact $E_{6}$ algebra, whose twenty-six non-compact generators are themselves represented by traceless Jordan matrices, and whose fifty-two compact generators close on $F_{4}$ [12]. Moreover these latter generators act as derivations on the Jordan matrices [12]. A distinguished set of derivations, corresponding to a $D_{4}$ subalgebra, are those which annihilate the diagonal elements 
$e_{i}$ :

$$
\delta e_{i}=0
$$

Put another way, the action of the $E_{6}$ generators may be taken to be

$$
\begin{aligned}
\delta_{X} J & =X \circ J, \quad \operatorname{Tr} X=0, \\
\delta_{X, Y} J & =X \circ(Y \circ J)-Y \circ(X \circ J), \quad \operatorname{Tr} X=\operatorname{Tr} Y=0,
\end{aligned}
$$

and this is the way we shall like to think of them whenever it is necessary to do so. The fact that (1.13) is actually a derivation, i.e.

$$
\delta(A \circ B)=\delta A \circ B+A \circ \delta B,
$$

follows from the associator identity

$$
(X, Y, W \circ Z)+(W, Y, Z \circ X)+(Z, Y, X \circ W)=0,
$$

where,

$$
(X, Y, Z)=X \circ(Y \circ Z)-(X \circ Y) \circ Z .
$$

Equation (1.14) itself is a direct consequence of Eq. (1.2). However, the fact that any derivation has the form (1.13) is a theorem [12].

The $F_{4}$ subalgebra leaves invariant the quadratic form

$$
Q=\operatorname{Tr}(X \circ Y),
$$

while the full $E_{6}$ has a cubic invariant:

$$
C=\operatorname{Tr}(X \circ(Y \times Z)),
$$

where the Freudenthal product [13] is defined to be

$$
Y \times Z=Y \circ Z-\frac{1}{2} Y \operatorname{Tr} Z-\frac{1}{2} Z \operatorname{Tr} Y+\frac{1}{2}(\operatorname{Tr} Y \operatorname{Tr} Z-\operatorname{Tr}(Y \circ Z)) 1 .
$$

It is interesting to note that the non-compact $E_{6}$ also contains as a non-compact subalgebra $s o(9,1)$, which like the compact subalgebra $F_{4}$, contains $D_{4}$. Note, the quadratic form is not $s o(9,1)$ invariant.

Goddard, Nahm, Olive, Ruegg and Schwimmer (GNORS) [5] have demonstrated that part of the Jordan product, Eq. (1.10), can be represented by vertex operators defined on the shortest (i.e. length squared one) vector, spinor or conjugate spinor weights of $D_{4}$. This fact is interesting since these particular vertex operators also occur in the superstring [22] viewed from the light-cone (or transverse) gauge. They represent the Ramond [18] or Neveu-Schwarz [19] fields, and the fermion emission vertex or its conjugate [20], respectively. GNORS did not, however, represent the rest of the algebra, Eqs. (1.7)-(1.9), involving the diagonal units $e_{i}$, and it is tempting to speculate that to do so must involve the vertex operators of the covariant superstring. These, in turn, inevitably involve factors containing BRST ghosts [21]. We shall demonstrate the sense in which we have found this speculation to be reasonable, and the sense in which the ghostly contributions are important for representing the diagonal units. It seems to us that the covariant lattice formulation of superstring models [21] may contain interesting structure, besides providing an approach to four dimensional string theories [23]. 
Actually, we have not found it possible to extend the GNORS representation to the remainder of the algebra in a straightforward way. Recall that their representation was based on the operator product of conformal weight one-half operators of the form,

$$
V(v, z) V(s, \omega) \sim(z-\omega)^{-1 / 2} V(\bar{s}, \omega),
$$

where $v \cdot s=-1 / 2$ and $v+s=\bar{s}$. One would then expect a leading square root singularity to occur in the operator products representing each of the non-zero terms in Eqs. (1.7)-(1.9). The major problem is how to represent the idempotents $e_{i}$. In fact, we have not found a satisfactory way to do so via vertex operators. On the other hand Eq. (1.8) suggests an alternative. Recall the operator product:

$$
l \cdot P(z) V(k, \omega) \sim\left(z-(\omega)^{-1} l \cdot k V(k, \omega),\right.
$$

where $P(z)=i \partial X(z)$. Equation (1.16) has the kind of structure required by Eq. (1.8) except for the singularity, which fails to match the singularity in Eq. (1.15). Unfortunately, even if the wrong singularity were permissible, we could not regard the idempotents as components of the momentum operator, since

$$
l \cdot P(z) k \cdot P(\omega) \sim l \cdot k(z-\omega)^{-2},
$$

which does not match Eq. (1.7).

One possibility is suggested by the schizophrenic rôle played by the Jordan elements themselves. As we have already mentioned, they can be regarded as a twenty-seven dimensional representation of $E_{6}$ and so might be represented as vertex operators defined on a set of 27 weights. We have tried that before and it does not by itself work [9]. Alternatively, the twenty-six traceless matrices are a subset of the generators of $E_{6}$, in the sense of Eq. (1.12). From that point of view, we might expect to represent these elements as vertex operators or momentum operators defined in terms of the $E_{6}$ roots. That also cannot by itself work. Apart from the objection raised above, the Frenkel-Kac-Segal (FKS) $[14,15]$ mechanism would force the operator products of these vertices to close within the $F_{4}$ subalgebra, whose generators are not themselves represented as Jordan elements, but rather as derivations.

We are led to suppose that the algebra cannot be represented in the normal operator product way; some modification is required. For example, it is known that the momentum operators alone corresponding to $N$ string degrees of freedom can represent the real Jordan algebras under a cross-bracket [17]. We have not discovered a way to a generalize this idea nor can we guess its relevance for the superstring. Another idea is to attempt to define a product using the action of $E_{6}$ on its twenty-seven dimensional representation. After all, if we are to append an "identity" operator to the set of twenty-six non-compact generators we ought to be able to define a "product" in which one factor is represented as a combination of vertex operators defined on the roots of $E_{6}$, and the other is a combination of vertex operators defined on the weights of $E_{6}$. What we need is a correspondence between the non-compact generators of $E_{6}$ and each of the basis elements of the exceptional Jordan algebra. As we shall see, it will be necessary to extend the root lattice by embedding it in a seven dimensional space, in order to define $\sum_{i} e_{i}$, the 
identity operator. Supposing this, we shall have a set of twenty-seven operators, denoted generically $L\left(J_{i}\right)$, with respect to which a general Jordan element $J=\sum j_{i} J_{i}$ is represented as

$$
L(J)=L\left(\sum j_{i} J_{i}\right)=\sum j_{i} L\left(J_{i}\right) .
$$

Similarly, we shall have a set of twenty-seven basis states defined on the weights (or, using the usual arguments of conformal field theory, vertex operators) $\left|J_{l}\right\rangle$ with respect to which a general Jordan element is expressed:

$$
|J\rangle=\left|\sum j_{i} J_{i}\right\rangle=\sum j_{i}\left|J_{i}\right\rangle \text {. }
$$

A useful observation is that among the twenty-seven states there is one, called the identity $|\mathbf{1}\rangle$, which is annihilated by all the generators of $F_{4}$ (put $J=1$ in Eq. (1.13)). In particular, $|\mathbf{1}\rangle$ is annihilated by the non-zero commutators of the non-compact $E_{6}$ generators $L(J)$. We shall find,

$$
\begin{aligned}
L(J)|\mathbf{1}\rangle & =|J\rangle, \\
L(J) L(K)|\mathbf{1}\rangle & =L(J)|K\rangle=|J \circ K\rangle=L(J \circ K)|\mathbf{1}\rangle,
\end{aligned}
$$

as a consequence of the detailed action of the vertex operators on the states. Note the product (1.20) is easily seen to be symmetric since

$$
|J \circ K\rangle-|K \circ J\rangle=[L(J), L(K)]|\mathbf{1}\rangle=0 .
$$

The Jordan identity becomes a statement about $F_{4}$ generators and can be checked directly without difficulty. At the level of the operators Eq. (1.2) becomes

$$
[L(X), L(X \circ X)]=0 .
$$

In terms of basis elements (1.22) may be written as

$$
\left[L\left(J_{i}\right), L\left(J_{i}^{\circ} J_{k}\right)\right]+\left[L\left(J_{k}\right), L\left(J_{i}^{\circ} J_{j}\right)\right]+\left[L\left(J_{i}\right), L\left(J_{k}^{\circ} J_{i}\right)\right]=0,
$$

and is reminiscent of a Bianchi identity. It is in the sense of Eq. (1.20) that the whole Jordan algebra is represented in terms of vertex operators. We shall find that the existence of an identity operator is closely related to the BRST ghosts.

In Sect. (4) we shall show how the same construction can be applied equally well for the other Jordan algebras, although there is no apparent relationship with any string theory.

\section{Jordan Elements as Vertex Operators and States}

$E_{6}$ Roots and Weights. The roots of $E_{6}$ may be expressed conveniently in terms of the roots and weights of $D_{4}$, and a set of (scaled) roots of $A_{2}$ :

$$
r=\left\{\begin{array}{l}
\left(\omega_{0}, 0\right) \\
\left(\omega_{i j}, \pm \alpha_{i j}\right) \quad i, j=1,2,3,
\end{array}\right.
$$

where $\omega_{0}$ is any root of $D_{4}$ (taken to satisfy $\left.\omega_{0}^{2}=2\right)$, and $\omega_{12}, \omega_{23}, \omega_{31}$ are any of the vector, spinor or conjugate spinor weights of $D_{4}$, respectively. The vectors 
$\alpha_{i j}$ are a scaled set of $A_{2}$ roots:

$$
\begin{aligned}
& \alpha_{i j}^{2}=1, \quad \alpha_{i j} \cdot \alpha_{j k}=-\frac{1}{2} \quad i \neq k, \\
& \alpha_{12}+\alpha_{23}+\alpha_{31}=0 .
\end{aligned}
$$

Recalling the strategy outlined in the introduction, we shall regard the scaled $A_{2}$ roots as embedded in a three dimensional space and able to be expressed in terms of a "spray" of three real linearly independent vectors $v_{i}, i=1,2,3$, entirely orthogonal to the $D_{4}$ weight space. Thus,

$$
\alpha_{i j}=v_{i}-v_{j}
$$

where,

$$
v_{i} \cdot v_{j}= \begin{cases}1 / 2+\beta & i=j \\ \beta & i \neq j .\end{cases}
$$

Note, (2.3) and (2.4) imply (2.2) with no restriction on $\beta$. In addition

$$
\sum v_{i} \cdot r=0
$$

for any $E_{6}$ root $r$.

The weights of the 27 of $E_{6}$ may be expressed similarly,

$$
w=\left\{\begin{array}{l}
\left(0, \lambda_{i i}\right) \\
\left(\omega_{i j}, \lambda_{i j}\right) \quad i=1,2,3,
\end{array}\right.
$$

where the vectors $\lambda_{i j}$ are defined to be,

$$
\hat{\lambda}_{i j}=v_{i}+v_{j} \text {. }
$$

Since the vectors $v_{i}$ are, for general $\beta(\beta \neq-1 / 6)$ linearly independent, the weights (2.5) are not symmetrically placed relative to the origin. Nevertheless, the inner products between roots and weights are as they should be; that is,

$$
\hat{i}_{i j} \cdot \alpha_{j k}= \begin{cases}0 & i=k \\ \pm 1 / 2 & \text { otherwise }\end{cases}
$$

or,

$$
r \cdot w=0 \text { or } \pm 1
$$

The normalizations of the weights $w$ depends on $\beta$,

$$
\begin{aligned}
w^{2} & =2+4 \beta, \\
w \cdot w^{\prime} & =4 \beta \text { or } \quad 1+4 \beta .
\end{aligned}
$$

Hence, the weights are integral provided $4 \beta$ is a integer. As we shall see, particularly interesting cases occur for $\beta=0$ or $-1 / 4$. The signature of the metric in the subspace spanned by the $v_{i}$ is also dependent upon $\beta$. In fact, the eigenvalues of the metric are always

$$
\frac{1}{2}+N \beta, \quad \frac{1}{2}(N-1 \text { times })
$$

for any $N$ dimensional spray. Thus, for $\beta=0$ the metric is Euclidean as expected, 
but for $\beta=-1 / 4$ the space is Minkowski, provided $N \geqq 3$. For $N=3$ the metric has eigenvalues,

$$
\frac{1}{2}, \frac{1}{2},-\frac{1}{4},
$$

and the vectors $\lambda_{i j}$ are each light-like. In the latter case, the $E_{6}$ roots and weights have been embedded in a seven dimensional Minkowski space; a fact which certainly suggests a connection with the superstring vertices and ghosts in their bosonized form [21]. In the former case, $\beta=0$, the weights and roots together are roots of $E_{7}$. Finally, there is another set of twenty-seven weights corresponding to (2.5), with the signs of the $v$ 's reversed:

$$
\bar{w}=\left\{\begin{array}{l}
\left(0,-\lambda_{i i}\right) \\
\left(\omega_{i j},-\lambda_{i j}\right) \quad i=1,2,3,
\end{array}\right.
$$

$E_{6}$ and $F_{4}$ Vertex Operators. The $E_{6}$ algebra can be represented in terms of vertex operators using the FKS construction [16]. The vertex operators are defined using the string field,

$$
X^{a}(z)=q^{a}-i p^{a} \ln z+i \sum_{\substack{n \in Z \\ \neq 0}} \frac{\alpha_{n}^{a}}{n} z^{-n}, \quad a=1, \ldots, 6,
$$

and the roots, $r,(2.1)$. Explicitly,

$$
V(r)=\oint \frac{d z}{2 \pi i} V(r, z)
$$

where

$$
V(r, z)=: \exp i r \cdot X(z): C(r)
$$

and the factors $C(r)$ are special functions of momentum, necessary for the correct insertion of the appropriate cocycles. Thus setting

$$
\hat{C}(r)=e^{i r \cdot q} C(r)
$$

we have the (associative) product,

$$
\widehat{C}(r) \hat{C}(s)=(-)^{r \cdot s} \widehat{C}(s) \hat{C}(r)=\varepsilon(r, s) \hat{C}(r+s),
$$

implying that $\varepsilon$ is a 2-cocycle:

$$
\varepsilon(r, s) \varepsilon(r+s, t)=\varepsilon(r, s+t) \varepsilon(s, t)
$$

and

$$
S(r, s)=\frac{\varepsilon(r, s)}{\varepsilon(s, r)}\left(=(-)^{r \cdot s}\right)
$$

The symmetry factor in brackets is appropriate for the $E_{6}$ roots, but not necessarily for the weights. We shall also assume $\widehat{C}(r) \hat{C}(-r)=1, \hat{C}(-r)=\hat{C}^{\dagger}(r)$, which amounts to a partial "gauge" fixing of the cocycle. 
In terms of the vertex operators and momenta $p^{a}$, the $E_{6}$ algebra is

$$
\begin{aligned}
& {\left[p^{a}, p^{b}\right]=0, \quad\left[p^{a}, V(r)\right]=r^{a} V(r),} \\
& {[V(r), V(s)]=\left\{\begin{array}{ll}
0 & r \cdot s \neq-1,-2 \\
\therefore(r, s) V(r+s) & r \cdot s=-1 \\
r \cdot p & r \cdot s=-2
\end{array} .\right.}
\end{aligned}
$$

Equation (2.11) refers only to the six components of the string field appropriate to the six-dimensional Euclidean lattice spanned by the roots of $E_{6}$. However, as we have indicated in the introduction, we shall need an extra additional component in order to be able to accommodate the Jordan product. Using the extra component, the twenty-seven states corresponding to the weights in (2.5) are,

$$
|w\rangle=e^{i w q}|0\rangle
$$

and we note the action of the vertex operators:

$$
\begin{aligned}
p|w\rangle & =w|w\rangle, \\
V(r)|w\rangle & = \begin{cases}0 & r \cdot w \neq-1 \\
\varepsilon(r, w)|w+r\rangle & r \cdot w=-1 .\end{cases}
\end{aligned}
$$

In the latter part of (2.18) we have assumed the extension of the cocycle and symmetry factor, $S(x, y)=\varepsilon(x, y) / \varepsilon(y, x)$, to the roots and weights in the seven dimensional space.

Our next task is to divide the $E_{6}$ generators into a set closing on $F_{4}$, and the other twenty-six non-compact generators. Fortunately, this is easy to do with the aid of an involution automorphism, or second order "twist," of the roots. The appropriate automorphism, $\sigma$, has the effect of interchanging $\omega_{i j}+\alpha_{i j}$ and $\omega_{i j}-\alpha_{i j}$, but leaving the $D_{4}$ roots, $\omega_{0}$ unaffected. That is

$$
\sigma\left(\omega_{i j} \pm \alpha_{i j}\right)=\omega_{i j} \mp \alpha_{i j}, \quad \sigma\left(\omega_{0}\right)=\omega_{0} .
$$

Lifting this automorphism to the algebra, it is clear that four dimensions worth of momenta (the $D_{4}$ Cartan subalgebra), is invariant, but the remainder, $v_{i} \cdot p$, changes sign. It is equally clear that while the vertex operators corresponding to the $D_{4}$ roots are invariant, there will be combinations of the twenty-four pairs of vertex operators $V\left(\omega_{i j} \pm x_{i j}\right)$ which are not. In other words the twenty-seven operators we are after, will be the twenty-four non-invariant combinations of vertex operators, and the momenta in the space spanned by the "spray" of vectors $v_{i}$, (2.3), (2.4). More specifically, we must ensure the operators representing the Jordan elements are hermitian. Thus, we shall write

$$
\begin{aligned}
U^{ \pm}\left(\omega_{\imath j}\right)= & \frac{1}{2}\left[a_{\omega_{1,},} V\left(\omega_{i j}+\alpha_{i j}\right) \pm b_{\omega_{l j},} V\left(\omega_{i j}-\alpha_{i j}\right)\right. \\
& \left.+b_{\omega_{1},}^{*} V\left(-\omega_{i j}+\alpha_{i j}\right) \pm a_{\omega_{l,}}^{*} V\left(-\omega_{i j}-\alpha_{i j}\right)\right],
\end{aligned}
$$

where the plus sign corresponds to the twist non-invariant generators, the minus sign to the twist invariant generators. The twist lifts into the $E_{6}$ algebra via,

$$
\sigma(V(r))=\sigma_{r} V(\sigma(r))
$$


where

$$
\begin{gathered}
\sigma_{\sigma(r)} \sigma_{r}=1, \quad \sigma_{\sigma(r)}=\sigma_{r}^{*}, \\
\sigma_{x} \sigma_{v} \varepsilon(\sigma(x), \sigma(y))=\sigma_{x+y} \varepsilon(x, y) .
\end{gathered}
$$

To ensure the twist invariant combination is the expected one, we also have

$$
a_{\left(\omega_{1 j}\right.}=-b_{\omega_{1 j}} \sigma_{\omega_{1 j}-x_{1 j}}
$$

The twist invariant combination must annihilate the state $|\mathbf{1}\rangle$, which we naturally define to be

$$
|\mathbf{1}\rangle=\sum_{i}\left|e_{i}\right\rangle, \quad\left|e_{i}\right\rangle=\left|2 v_{\imath}\right\rangle=\left|\lambda_{i i}\right\rangle,
$$

and provides a further relationship between the coefficients $a_{\omega_{2}}$, and $b_{\omega_{1},}$. Specifically, it is,

$$
a_{\omega_{i},} \varepsilon\left(\omega_{i j}+\alpha_{i j}, \hat{\lambda}_{l j}\right)=b_{\omega_{2 j}} \varepsilon\left(\omega_{i j}-\alpha_{i j}, \lambda_{i i}\right) .
$$

Equations (2.23) and (2.25) together provide an expression for the phase, $\sigma_{r}$, occurring in Eq. (2.21) which must be consistent with Eq. (2.22). Indeed, using (2.23), (2.25) and the cocycle condition (2.15), Eq. (2.22) reduces to a correct statement about the symmetry factor (making use of the bilinearity of the extended symmetry factor).

Equation (2.20) supply twenty-four of the Jordan operators. The other three are momentum contributions of the form

$$
L\left(e_{i}\right)=\chi_{i} \cdot p,
$$

where the vectors $\chi_{i}$ are linear combinations of the three $v$ 's, designed so that

$$
L\left(e_{i}\right)\left|e_{j}\right\rangle=\chi_{i} \cdot p\left|\lambda_{j j}\right\rangle=2 \chi_{i} \cdot v_{j}\left|e_{j}\right\rangle=\delta_{i j}\left|e_{j}\right\rangle .
$$

Thus,

$$
\chi_{i} \cdot v_{j}=\frac{1}{2} \delta_{i j} \Rightarrow \chi_{l}=v_{i}-\frac{\beta}{1 / 2+N \beta} \sum_{i} v_{i}
$$

and the identity operator $L(\mathbf{1})$ is given by

$$
L(\mathbf{1})=\sum_{i} L\left(e_{i}\right)=\frac{1 / 2}{1 / 2+N \beta} \sum_{i} v_{i} \cdot p=s \cdot p .
$$

It is easy to check that $L(\mathbb{1})$ commutes with every $E_{6}$ generator, and hence everything in the list (2.20). Moreover, its action on the twenty-seven weights is as it should be, $L(1)|w\rangle=|w\rangle$. These facts follow from the observations

$$
s \cdot r=0, \quad s \cdot w=1,
$$

where $r$ may be any $E_{6}$ root, and $w$ is any of the $\mathbf{2 7}$ (not $27^{\prime}$ ) weights, Eqs. (2.5), (2.6).

We note also, the operators $L\left(e_{i}\right)$ satisfy

$$
L\left(e_{i}\right)|\mathbf{1}\rangle=\sum_{j} L\left(e_{i}\right)\left|e_{j}\right\rangle=\left|e_{\imath}\right\rangle,
$$

as they ought. 
Finally, we end this section by examining the action of each of the twenty-four twist non-invariant operators $(2.20)$ on the identity state:

$$
\begin{aligned}
U^{+}\left(\omega_{i j}\right)|\mathbf{1}\rangle= & a_{\omega_{i j}} \varepsilon\left(\omega_{i j}+\alpha_{i j}, \lambda_{j j}\right)\left|\omega_{i j}+\lambda_{i j}\right\rangle \\
& +b_{\omega_{1 j}}^{*} \varepsilon\left(-\omega_{i j}+\alpha_{i j}, \lambda_{j j}\right)\left|-\omega_{i j}+\lambda_{i j}\right\rangle,
\end{aligned}
$$

where we have made a partial use of Eq. (2.25). It is easy to check,

$$
U^{+}\left(\omega_{i j}\right)\left(U^{+}\left(\omega_{t j}\right)|\mathbf{1}\rangle\right)=\left|a_{\omega_{i j}}\right|^{2}\left(\left|\lambda_{l i}\right\rangle+\left|\lambda_{j j}\right\rangle\right)
$$

which implies the coefficient $a_{\omega_{1}}$ is a phase, in order to guarantee the product (1.9). Again, (2.32) follows from the cocycle condition and the properties of the $E_{6}$ symmetry factor.

\section{The Jordan Algebra}

For each of the special weights of $D_{4}$, we have defined a hermitian operator, (2.20), and a corresponding state (2.31), each of which depends upon a phase, $a_{\omega_{1}}$, yet to be determined. If we were to follow as closely as possible the manipulations of GNORS we should be forced to select these phases, and a representation of the cocycles, so that each hermitian operator, corresponding to a certain triple of weights selected from $\omega_{12}, \omega_{23}, \omega_{31}$, would correspond to an off-diagonal Jordan matrix containing either unity or one of the octonion units as elements. Then the algebra product (1.10) can be checked directly.

An alternative, and more general, strategy is to define the product in terms of the operators (2.20) and their action on the weight states, and check the Jordan identity directly without any specific reference to the octonions, or any particular choice for the cocycle. To facilitate this check we shall change notation slightly to incorporate the unspecified phases. Thus, we write

$$
L\left(a, \Omega_{i j}\right) \equiv U^{+}\left(\omega_{i j}\right), \quad|a|^{2}=1,
$$

where by convention we shall suppose,

$$
\Omega_{i j}=\omega_{i j}+\alpha_{i j}, \quad \Omega_{j i}=\omega_{i j}-\alpha_{i j}=\omega_{i j}+\alpha_{j i},
$$

where $\omega_{i j}$ is any of the weights in the $i j^{\text {th }}$ weight set, and so,

$$
L\left(a,-\Omega_{i j}\right)=L\left(a^{*}, \Omega_{i j}\right), \quad L\left(a,-\Omega_{j i}\right)=L\left(-a^{*} \sigma_{\omega_{i j}}, \Omega_{i j}\right) .
$$

Notice, there are eight independent operators for each pair $(i j)$, as there should be. Similarly, the state corresponding to $L\left(a, \Omega_{i j}\right)$ is denoted by

$$
L\left(a, \Omega_{i j}\right)|\mathbf{1}\rangle=\left|a, \Omega_{i j}\right\rangle \equiv a \varepsilon\left(\Omega_{i j}, \lambda_{j j}\right)\left|\omega_{i j}+\lambda_{i j}\right\rangle+b^{*} \varepsilon\left(-\Omega_{j i}, \lambda_{j j}\right)\left|-\omega_{i j}+\lambda_{i j}\right\rangle .
$$

Then, the Jordan algebra is defined in terms of the operators $L\left(a, \Omega_{i j}\right)$ and $L\left(e_{i}\right)$, with the basic products:

$$
\begin{gathered}
L\left(e_{i}\right)\left|e_{j}\right\rangle=L\left(e_{i}\right) L\left(e_{j}\right)|\mathbf{1}\rangle=\delta_{i j} L\left(e_{j}\right)|\mathbf{1}\rangle=L\left(e_{i} \circ e_{j}\right)|\mathbb{1}\rangle, \\
L\left(e_{l}\right) L\left(a, \Omega_{j k}\right)|\mathbf{1}\rangle=\psi_{i} \cdot p\left|a, \Omega_{\jmath k}\right\rangle= \begin{cases}0 & i \neq j \text { or } k \\
1 / 2\left|a, \Omega_{j k}\right\rangle & i=j \text { or } k\end{cases}
\end{gathered}
$$




$$
\begin{aligned}
& = \begin{cases}0 & i \neq j \text { or } k \\
1 / 2 L\left(a, \Omega_{j k}\right)|\mathbf{1}\rangle & i=j \text { or } k,\end{cases} \\
& L\left(a, \Omega_{i j}\right) L\left(a^{\prime}, \Omega_{j k}^{\prime}|\mathbf{1}\rangle=L\left(a, \Omega_{i j}\right)\left|a^{\prime}, \Omega_{j k}^{\prime}\right\rangle=\left|a^{\prime \prime}, \Omega_{i k}^{\prime \prime}\right\rangle\right. \\
& =L\left(a^{\prime \prime}, \Omega_{i k}^{\prime \prime}\right)|\mathbf{1}\rangle \quad i \neq k,
\end{aligned}
$$

with $a^{\prime \prime}=a a^{\prime} \varepsilon\left(\Omega_{i j}, \Omega_{j k}^{\prime}\right), \Omega_{i k}^{\prime \prime}=\Omega_{i j}+\Omega_{j k}^{\prime}$, provided $\omega_{i j} \cdot \omega_{j k}^{\prime}=-1 / 2$ (we need not consider the case when $\omega_{i j} \cdot \omega_{j k}^{\prime}=1 / 2$ separately since we can re-express it above using the relations (3.2)),

$$
L\left(a, \Omega_{i j}\right) L\left(a^{\prime}, \Omega_{i j}^{\prime}\right)|\mathbf{1}\rangle= \begin{cases}0 & \omega_{i j} \cdot \omega_{i j}^{\prime}=0 \\ \operatorname{Re}\left(a^{*} a^{\prime}\right)\left(L\left(e_{i}\right)+L\left(e_{j}\right)\right)|\mathbf{1}\rangle & \Omega=\Omega_{i j}^{\prime} \\ \operatorname{Re}\left(a a^{\prime}\right)\left(L\left(e_{i}\right)+L\left(e_{j}\right)\right)|\mathbf{1}\rangle & \Omega_{i j}=-\Omega_{i j}^{\prime} .\end{cases}
$$

As we remarked before, each of these products is actually symmetric since the commutator of any pair of L's is an $F_{4}$ generator which annihilates the identity state by construction.

To establish that Eqs. (3.4)- (3.7) do indeed yield a Jordan product, the identity (1.23), equivalent to the Jordan identity, must be checked. This is not difficult. Basically, there are four different possibilities.

(i) Three diagonal units $L\left(e_{i}\right), L\left(e_{j}\right), L\left(e_{k}\right)$; each term in the identity is trivially zero.

(ii) Two diagonal units, $L\left(e_{i}\right), L\left(e_{j}\right)$ and one off diagonal unit $L\left(a, \Omega_{k l}\right)$; the identity is trivial when $i$ and $j$ match $k$ and $l$, respectively (or vice versa), in which case useful facts are

$$
\begin{aligned}
& {\left[\chi_{i} \cdot p, U^{+}\left(\omega_{i j}\right)\right]=\frac{1}{2} U^{-}\left(\omega_{i j}\right),} \\
& {\left[\chi_{j} \cdot p, U^{+}\left(\omega_{i j}\right)\right]=-\frac{1}{2} U^{-}\left(\omega_{i j}\right),}
\end{aligned}
$$

from which the identity follows, using also (3.5).

(iii) One diagonal unit, $L\left(e_{l}\right)$, and a pair of off diagonal units $L\left(a, \Omega_{k l}\right), L\left(a^{\prime}, \Omega_{n m}^{\prime}\right)$; the identity requires checking when $l=m$ and $i=$ either $l$ or $n$. It follows from (3.8), using (3.5) and (3.6) to define the products of the various units.

(iv) Three diagonal units, $L\left(a, \Omega_{i j}\right), L\left(a^{\prime}, \Omega_{k l}^{\prime}\right), L\left(a^{\prime \prime}, \Omega_{n m}^{\prime \prime}\right)$; since the pairs of labels may only be chosen from the set $\{(1,2),(2,3),(3,1)\}$ there are three possibilities. If $(i, j)=(k, l)=(n, m)$ the identity follows from (3.7) and (3.8). If $(i, j)=(k, l) \neq(n, m)$ the identity follows from (3.6), (3.7) and (3.8). If the three labels are different the identity is trivially satisfied unless $\omega+\omega^{\prime}+\omega^{\prime \prime}=0$. In that case, it is useful to note

$$
\left[L\left(a, \Omega_{i}\right), L\left(a^{\prime} a^{\prime \prime} \varepsilon\left(\Omega_{j k}^{\prime}, \Omega_{k i}^{\prime \prime}\right),-\Omega_{i j}\right)\right]=\operatorname{Im}\left(a a^{\prime} a^{\prime \prime} \varepsilon\left(\Omega_{j k}^{\prime}, \Omega_{k i}^{\prime \prime}\right)\right) \omega_{i j} \cdot p,
$$

and

$$
\varepsilon\left(\Omega_{i j}, \Omega_{j k}^{\prime}\right)=\varepsilon\left(\Omega_{j k}^{\prime}, \Omega_{k i}^{\prime \prime}\right)=\varepsilon\left(\Omega_{k i}^{\prime \prime}, \Omega_{i j}\right),
$$

from which the identity follows. Equation (3.9) is a direct consequence of the algebra of the vertex operators defined in (2.20), and the condition (2.25).

\section{Local Vertex Operators}

To each $E_{6}$ generator, there corresponds a vertex operator. To each weight state we also have a corresponding vertex operator $V(w, z)$. However, although the latter 
have local operator products with respect to the former, they will not in general be local with respect to each other. Special cases occur when $4 \beta$ is an integer, as we have already mentioned. We shall explore the implications of the choices $4 \beta=0,-1$ in this section.

We have seen in what sense any twenty-seven dimensional representation of $E_{6}$ generates a Jordan algebra provided the roots and weights are non-trivially embedded in a seven dimensional space. The leading term in the operator product corresponding to these weights is:

$$
V(w, z) V\left(w^{\prime}, \omega\right) \sim \varepsilon\left(w, w^{\prime}\right)(z-\omega)^{w \cdot w^{\prime}} V\left(w+w^{\prime}, \omega\right) .
$$

The relations (2.8) imply these operator products are non-local unless $4 \beta$ is an integer. An obvious choice is to take $4 \beta=0$, in which case the right-hand side of Eq. (4.1) is never singular. Moreover, in that case, the length of any weight is $\sqrt{2}$, the same as a root. The metric in the space spanned by the weights is Euclidean. In fact, these weights, together with the $27^{\prime}$, Eq. (2.10), contribute the set of roots which must be added to the $E_{6}$ roots to yield the roots of $E_{7}$. The vector $s,(2.28)$, used to define the identity of the Jordan algebra is a special $E_{7}$ weight corresponding to the label:

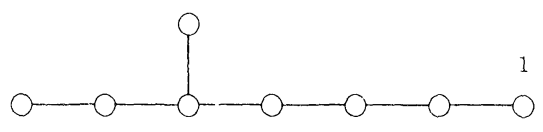

In other words, the highest weights of the $\mathbf{5 6}$ representation of $E_{7}$. These statements match similar statements made in ref. [11].

Another choice is $4 \beta=-1$. The operator products (4.1) are now, in general, singular. Also, the length of any weight is unity, and so we would expect the vertex operators to be fermionic in character. Indeed, the naive conformal weight of $V(w, z)$ is $w^{2} / 2=1 / 2$. This is certainly their conformal weight relative to the standard energy-momentum operator:

$$
T(z)=\frac{1}{2} P(z) \cdot P(z)=\sum L_{n} z^{-n-2} .
$$

In addition, the appropriate choice for the symmetry factor is

$$
S\left(w, \omega^{\prime}\right)=(-)^{w \cdot w^{\prime}+w^{2} w^{\prime 2}},
$$

the bilinear choice which guarantees that vectors of odd (even) length correspond to fermionic (bosonic) vertex operators. (The choice (4.3) is in accord with all our previous arguments involving symmetry factors.) However, as we pointed out briefly, earlier, these weights are not standard since the metric in the space spanned by them has signature $\left((+)^{6},(-)\right)$. Indeed the vectors $\lambda_{i j}$ are light-like, while

$$
s^{2}=\left(-2 \sum v_{i}\right)^{2}=-3 .
$$

To gain a better understanding of the meaning of the vector $s$ we notice that if we shift the Virasoro generators (4.2) by an amount proportional to $s$ :

$$
L_{n}^{\prime}=L_{n}+\frac{1}{2}(n+1) s \cdot \alpha_{n},
$$


the central charge is now given by

$$
c=\frac{D}{12}-\frac{s^{2}}{4}=\frac{7}{12}+\frac{3}{4}=\frac{16}{12} \text {. }
$$

Also, with respect to the new Virasoro generators the vertex $V(w, z)$ has conformal weight

$$
\Delta_{w}=\frac{1}{2} w \cdot(w+s),
$$

precisely unity since $w \cdot s=1$, Eq. (2.29). We also remark, the anomaly (4.6) is exactly appropriate to be cancelled by an ordinary bosonic string anomaly in ten dimensions $(D / 12-26 / 12=-16 / 12)$. In other words, the choice $4 \beta=-1$ yields a set of vertex operators which correctly include the ghostly contributions of the covariant superstring vertices. Moreover, the identity operator in the Jordan algebra is determined by the background "ghost charge," as is easily checked on comparing (4.7) with the standard formulae for the conformal weights of the super-conformal ghosts of the superstring. This seems to us to be a suprising and mysterious connection.

By choosing a specific form of basis we may write

$$
\begin{aligned}
& v_{1}=(0,0,-1 / 2), \\
& v_{2}=(-1 / 2,-1 / 2,1 / 2), \\
& v_{3}=(1 / 2,-1 / 2,1 / 2),
\end{aligned}
$$

where the middle entry refers to the component which contributes with a negative sign in the inner product. The middle entry refers, in the usual interpretation, to the " $\phi$ " ghost, the last entry to the " $\chi$ " ghost in the notation of ref. [21]. Thus, the original $E_{6}$ weights are explicitly written:

$$
\begin{gathered}
(\mathbf{0}, 0,0,-1), \\
\left\{\begin{array}{l}
(\mathbf{0}, \pm 1,-1,1) \\
(\mathbf{v}, 0,-1,1)
\end{array}\right. \\
\left\{\begin{array}{l}
(\mathbf{s}, 1 / 2,-1 / 2,0) \\
(\mathbf{c},-1 / 2,-1 / 2,0),
\end{array}\right.
\end{gathered}
$$

where $\mathbf{v}, \mathbf{s}, \mathbf{C}$ are the $D_{4}$ fundamental weights. The bracketed sets of weights are gathered together as $s o(9,1)$ weights, a vector and a chiral spinor, respectively. The vector $s$ is seen to be

$$
s=-2 \sum v_{i}=(\mathbf{0}, 0,2,-1),
$$

and so according to Eq. (4.7) the conformal dimension of a vertex operator associated with an arbitrary vector of the form $w=\left(\lambda, q_{\phi}, q_{\chi}\right)$, where $\lambda$ is an $\operatorname{so}(9,1)$ weight, is given by

$$
\Delta_{w}=\frac{1}{2} \lambda^{2}-\frac{1}{2} q_{\phi}\left(q_{\phi}+2\right)+\frac{1}{2} q_{\chi}\left(q_{\chi}-1\right)
$$

just as it should be, verifying our claim that the identity of the Jordan algebra is represented by the background ghost charge. 
Corresponding to the basis (4.9), the $E_{6}$ roots are:

$$
\begin{aligned}
& \left(\omega_{0}, 0,0,0\right), \\
& (\mathbf{v}, \pm(-1,0,0)), \\
& (\mathbf{s}, \pm(1 / 2,-1 / 2,1)), \\
& (\mathbf{c}, \pm(1 / 2,1 / 2,-1)),
\end{aligned}
$$

and it is natural to ask if they (and the weights (4.9)) may be regarded as elements of a seven dimensional Lorentzian lattice extending the $E_{6}$ root lattice. Such a lattice is not associated with the $E_{7}$ Dynkin diagram, which defines a Euclidean lattice. Nor is it merely the affine $E_{6}$ Dynkin diagram. That extends the $E_{6}$ lattice by appending multiples of a light-like vector, whereas our lattice contains vectors whose squared lengths are actually negative. In other words, the lattice we are seeking is hyperbolic, rank 7, and there are only a few of these $[24,25]$. In fact, the possibilities correspond to the diagrams,

(a)

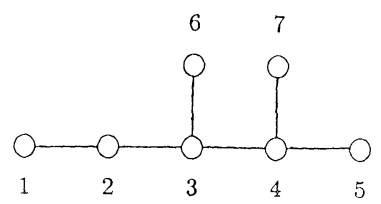

(b)

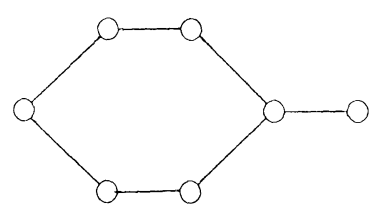

(c)

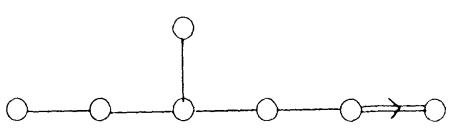

and actually, it is the first, (a), which is the appropriate one for us, the others requiring irrational points relative to (4.11). We have no explanation for this observation. In this case, the vector $-s / 2$ represents the fundamental weight corresponding to

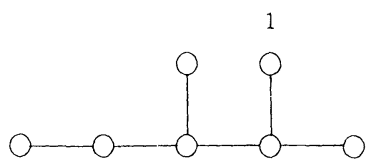

Selecting a set of simple roots, corresponding to the labelling in (a) above we could take,

$$
\begin{aligned}
& r_{1}:(-1,0,0,0,1,0,0) \\
& r_{2}:(1,-1,0,0,0,0,0) \\
& r_{3}:(0,1,-1,0,0,0,0) \\
& r_{4}:(0,0,1,1,0,0,0) \\
& r_{5}:(-1 / 2,-1 / 2,-1 / 2,-1 / 2,-1 / 2,1 / 2,-1) \\
& r_{6}:(0,0,1,-1,0,0,0),
\end{aligned}
$$


in which case the seventh root turns out to be,

$$
r_{7}:(-1 / 2,-1 / 2,-1 / 2,-1 / 2,-1 / 2,1 / 2,1) \text {. }
$$

All but $r_{7}$ are orthogonal to $s$, and

$$
r_{7} \cdot s=-2
$$

as claimed.

If we associate a vertex operator to every element of the lattice (a), those commuting with $L_{0}$, defined in (4.5), correspond to the zeroth moments of the $E_{6}$ operators. This fact demonstrates that the background ghost charge plays a rôle akin to a shift, breaking the hyperbolic algebra down to $E_{6}$. This rôle needs examining further. It seems not to have been studied previously since the " $\chi$ " ghost is mainly associated with the phenomenon of "picture changing," and otherwise ignored.

We end this section with one further remark. If we compute the operator product of vertex operators defined on the weights, we find

$$
\begin{aligned}
V\left(w_{27}, z\right) V\left(w_{27}^{\prime}, \omega\right) & \sim(z-\omega)^{w \cdot w^{\prime}} \varepsilon\left(w, w^{\prime}\right) V\left(w_{27^{\prime}}^{\prime \prime}-s, \omega\right), \\
V\left(w_{27^{\prime}}, z\right) V\left(w_{27^{\prime}}^{\prime}, \omega\right) & \sim(z-\omega)^{w \cdot w^{\prime}} \varepsilon\left(w, w^{\prime}\right) V\left(w_{27}^{\prime \prime}+s, \omega\right),
\end{aligned}
$$

where the subscripts $27,27^{\prime}$ refer to the two conjugate twenty-seven dimensional sets of weights. Equations (4.12) are related to the Freudenthal product, as we pointed out before [9]. Clearly, there are infinitely many copies of the twenty-seven dimensional representations, differing by $s$, obtained by computing operator products of a representation with itself. Since the vertex operators of the twenty-seven correspond to superstring vertex operators plus ghosts this replication of representations is highly suggestive of "picture changing" [21]. Of course, we cannot make a direct contact with the usual formalism because we have not discussed the bosonic part of the string. It would be interesting if the ghostly sector, and in particular picture changing, could be understood more algebraically than at present.

\section{Non-Exceptional Jordan Algebras}

With a suitably altered choice of roots and weights, the discussion of Sect. (2) can be taken over to discuss the other, non-exceptional Jordan algebras. In particular, the algebras playing the rôle in the quaternion $N \times N$ case (the other cases are special versions of this), analogous to $E_{6}$ and $F_{4}$, are a non-compact $s u(2 N)$ with a maximal compact subalgebra $s p(N)$. We may adopt the same notation as (2.1). However, in the new situation, the roots $\omega_{0}$ are roots of $s u(2)+\cdots+\operatorname{su}(2)$ ( $N$ times), the weights $\omega_{i j}$ are scaled roots of $s o(2 N)$ and the quantities $\alpha_{i j}$ are scaled roots of $\operatorname{su}(N)$ satisfying (2.2), together with

$$
\alpha_{i j} \cdot \alpha_{k l}=0, \text { if } i, j, k, l \text { different. }
$$

Specifically, we may take [5],

$$
\omega_{i j}=\frac{1}{\sqrt{2}}\left( \pm e_{i} \pm e_{j}\right),
$$


where $e_{i}, i=1, \ldots, N$, are orthonormal unit vectors. As before, we need a "spray" of vectors $v_{i}, i=1, \ldots, N$, such that

$$
\alpha_{i_{j}}=v_{i}-v_{j}
$$

and Eq. (2.2) holds. Equally, we may take over (2.5) with the definition

$$
\lambda_{i j}=v_{l}+v_{j}
$$

and the extra fact:

$$
\lambda_{i j} \cdot \alpha_{k l}=0, \text { if } i, j, k, l \text { different. }
$$

There are $N(2 N-1)$ weights, corresponding to the irreducible representation of $\operatorname{su}(2 N)$ with Dynkin label:

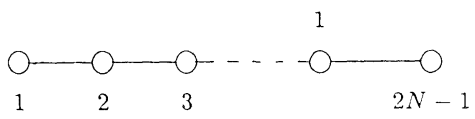

The weights are a reducible representation of $s p(N)$, decomposing into a singlet (the identity of the Jordan algebra), and an irreducible representation, corresponding to

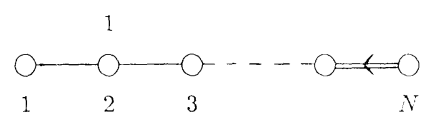

The discussion of the Jordan algebra follows the lines of the rest of the discussion in Sect. (2) rather closely. Indeed our notation was designed for this purpose.

As before, the vertex operators defined on the weights are bose or fermi depending on the choice $4 \beta=0,-1$, respectively. In the former case, the weights will be roots enhancing the $A_{(2 N-1)}$ Dynkin diagram to that of $D_{2 N}$, in which case $s$, the "shift," corresponds to a spinor weight. In the fermionic case, the signature of the space in which the weights lie is indefinite and given by (2.9). It is not clear what the appropriate Dynkin diagram might be for $2 N>10$. This is because the hyperbolic lattices have dimension $\leqq 10$. When $2 N=10$, the only possibility (containing $A_{9}$ as a sublattice) is $E_{10}$ :

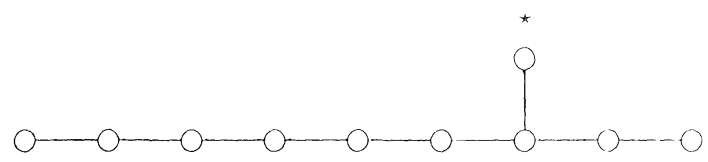

When $2 N=8$ or 6 , respectively, the possibilities (containing $A_{7}$ and $A_{5}$, respectively) are

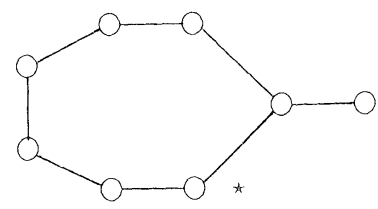


and

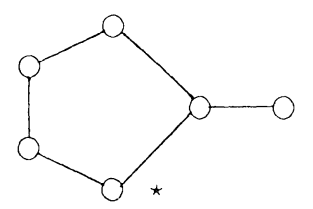

In each case, the "shift," $s$, has a non-zero inner product with the simple root labelled $(*)$.

Notice, the square of the shift vector is given by,

$$
s^{2}=-1-\frac{2}{N-2},
$$

and thus the conformal anomaly, corresponding to the shifted Virasoro algebra, is computed to be, (Eq. (4.7)),

$$
c=\frac{N}{12}\left(2+\frac{3}{N-2}\right) .
$$

Again, we may compare (5.6) with a bosonic anomaly and find that it cancels, for integer dimension, when $N=3,4,5,8$ corresponding to $D=11,12,11$ and 6 , respectively. We do not know if these "coincidences" are significant. It is however curious that the hyperbolic lattices mentioned above correspond to the first three special values of $N$, while the last, $N=8$, although it does not correspond to a hyperbolic lattice, is nevertheless an interesting one in the bose case. There, the extended lattice is $D_{16}$, enhanced by the shift vector to one of the self-dual lattices associated with the original heterotic string [26]! Actually, the $N=4$ case, in the bose situation, leads to the extended lattice $D_{8}$ enhanced by a spinor weight to the self-dual $E_{8}$ lattice.

\section{Discussion}

Our aim has been to discover a sense in which Jordan algebras may be represented by vertex operators. While we are unable to achieve this straightforwardly, we have discovered a way of defining a Jordan algebra which associates it rather closely with certain collections of vertex operators. Moreover, we have found two main ways of doing this, one of which is strikingly close to the superstring, in the sense that the vertex operators we need involve momenta lying in a non-Euclidean lattice. In addition, the non-Euclidean momenta, in the exceptional case, are exactly the same as those momenta corresponding to the bosonized fermion vertices of the superstring, taking account of their ghostly contributions. What we have not yet found is a Lagrangian starting point, although we feel that if the Jordan matrix is one of the basic ingredients, the Lagrangian will have to be cubic in order to accommodate the $S O(9,1)$ invariance (this is not the invariance group of any quadratic form involving Jordan matrices), and hence presumably it will have an $E_{6}$ or even greater symmetry. We are intrigued by the fact that the spacetime supersymmetry generator, which is after all a zero momentum vertex operator 
$[20,21]$, actually seems to belong naturally to an $E_{6}$ representation with fermionic character. As we have said, it seems to us that the usual decomposition of components into ghostly and non-ghostly pieces may be artificial and correspond to a choice of basis or "gauge." The usual choice of light-cone, or transverse, gauge is also expressed nicely in the Jordan language, via Eq. (1.11), restricting the derivations in the exceptional case to $D_{4}$.

Beyond this, we have yet to find a way to incorporate the bosonic part of the superstring in order to be able to explore further how supersymmetry and "picture changing" might fit in with the algebraic ideas we have discussed.

There is also a hint of other connections with hyperbolic lattices, remarked upon in Sect. (4), which we have hardly explored, but find intriguing and puzzling.

Acknowledgements. We are grateful to David Fairlie, Walter Troost and Toine Van Proeyen for comments and discussions. One of us (TJH) also wishes to thank the SERC for a research studentshıp.

\section{References}

1. Jordan, P., von Neumann, J., Wigner, E.: Ann. Math. 35. 29 (1934)

2. Gunaydin, M., Gursey. F.: J. Math. Phys. 14, 1651 (1973); Phys. Rev. D9, 83 (1974)

3. Gunaydin. M., Sierra, G., Townsend, P. Nucl. Phys. B242, 244 (1984)

4. Fairlie, D. B., Manogue, C.: Phys. Rev. D34, 1832 (1986)

5. Goddard, P., Nahm, W., Olive, D., Ruegg, H., Schwimmer, A.: Commun Math. Phys. 112, 385 (1987)

6. Sierra, G.: Class. Quantum Gravity 4, 227 (1987)

7. Gursey, F.: Mod. Phys Letts. A2, 967 (1987)

8. Foot, R., Joshi, G. C.: Phys. Letts. B199, 203 (1987)

9. Corrigan, E. Hollowood. T. J.: Phys. Letts B203, 47 (1988)

10. Gunaydin, M., Hyun, S. J.: preprint PSU/TH/44

11. Gunaydin, M.: preprint PSU/TH/46

12. Schafer, D.: Introduction to nonassociative algebras. New York: Academic Press 1966

13. Freudenthal, H.: Adv. Math. 1, 145 (1964)

14. Frenkel, I., Kac, V. G.: Invent. Math. 62. 23 (1980)

15. Segal, G.: Commun. Math Phys. 80, 301 (1981)

16. Goddard, P., Olive, D.: Int. J. Mod. Phys. A1, 303 (1986)

17. Frenkel, I., Lepowsky, J, Meurman, A.: A moonshine module for the monster. In: Vertex operators in mathematics and physics, MSRI 3. New York: Springer Berlin, Heidelberg, 1984

18. Ramond, P : Phys. Rev. D3, 2415 (1971)

19. Neveu, A., Schwarz, J. H.: Nucl. Phys. B31, 86 (1971)

20. Green, M. B., Schwarz, J. H.: Nucl. Phys. B181, 502 (1981)

21. Friedan, D., Martinec, E., Shenker, S.: Nucl. Phys. B271, 93 (1986)

22. Green, M. B., Schwarz, J. H., Witten, E.: Superstring theory. Cambridge: Cambridge Univ. Press 1987

23. See for example, Lerche, W., Schellekens, A. N.: The covariant construction of four dimensional strings, CERN-TH4925/87

24. Kac. V. G.: Infinite dimensional algebras. Boston, MA: Birkhauser 1984

25. Saclioglu, C.: String vertex operators and Dynkin diagrams for hyperbolic Kac-Moody algebras, CERN-TH4854/87

26. Gross, D. J., Harvey, J. A., Martinec, E.. Rohm, R.: Nucl. Phys. B256, 253 (1985)

Communicated by L. Alvarez-Gaumé

Received June 16, 1988 